\title{
Engineered plasmon focusing on functional gratings.
}

\author{
H.L. Offerhaus, B. van den Bergen and N.F. van Hulst
}

Applied Optics group, MESA ${ }^{+}$Institute for NanoTechnology, Department of Science \& Technology, University of Twente, The Netherlands http://nweb.tn.utwente.nl/ot/; H.L.Offerhaus@tn.utwente.nl

\begin{abstract}
We report on the engineering of plasmon propagation and focusing by dedicated curved gratings and noncollinear phasematching. Gratings were created on gold by focused ion beam milling and plasmons were measured using phase sensitive PSTM.

(C) 2005 Optical Society of America

OCIS codes: (240.6680) Surface plasmons; (070.2580)Fourier optics; (180.5810)Scanning microscopy
\end{abstract}

Surface plasmons can be exited resonantly when the plasmon k-vector matches the projected light k-vector [1]. A grating vector shifts the latter in an engineered continuous way. An appropriately shaped grating can ensure the creation of plasmons in a predefined direction, e.g. towards a common center [2]. For perpendicular illumination the required grating profile is a bulls-eye pattern, where the grating periodicity matches the plasmon resonance at the illumination wavelength. This grating is matched for converging plasmons as well as diverging plasmons. The degeneracy can be lifted by introduction of as asymmetry, such as an angled illumination. To achieve focusing of plasmons for angled incident illumination the grating has to add less k-vector before the focal point and more behind the focal point so that the grating pattern resembles cuts through angled cones or shifted circles (fig 1); corresponding to the Fourier transform of a circle in $\mathrm{k}$-space that has radius corresponding to the plasmon resonant $\mathrm{k}$-vector and is shifted from the center by an amount corresponding to the projected light vector.
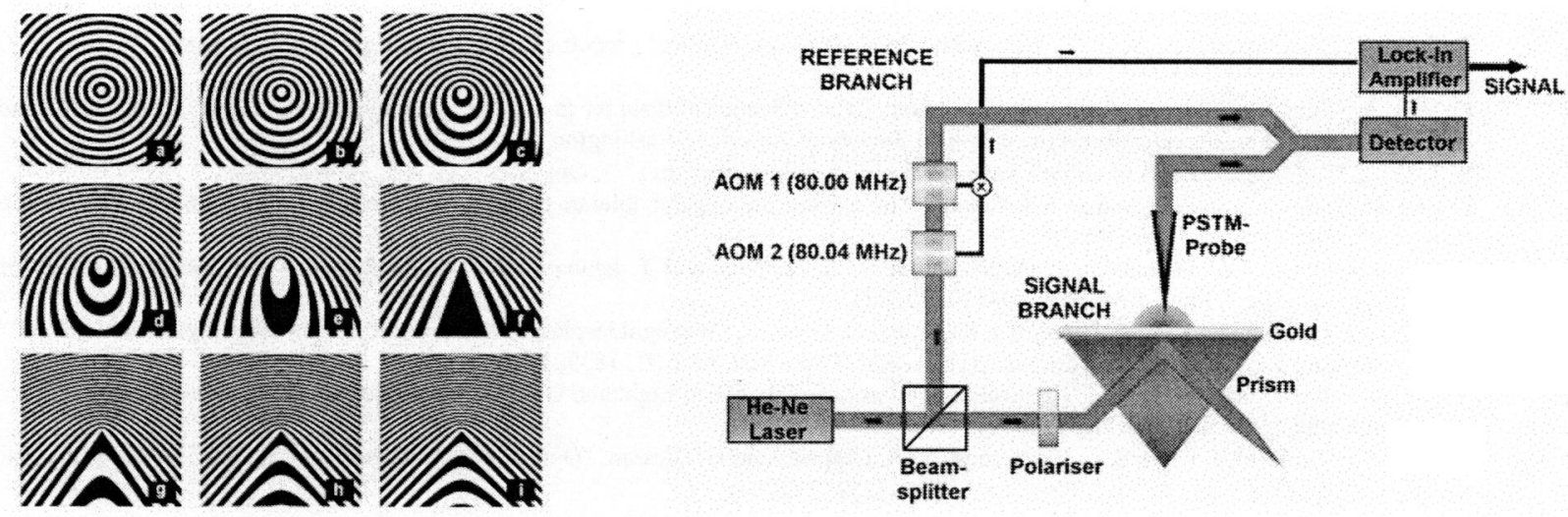

Figure 1 Grating profiles for focusing plasmons under increasingly angled illumination and PSTM setup. Figure 2 Phase sensitive Photon Scanning Tunneling Microcoscope (PSTM) setup. The grating profile is on the top side of the gold layer.

We present local probing of the plasmon propagation by phase sensitive PSTM (fig 2) of the field distribution on circles and stretched circles (fig 3,4), showing the focusing and directing of beams of plasmons. The gratings are created in a thin $(50 \mathrm{~nm})$ gold layer by Focused Ion Beam (FIB) milling.

Figure 3 shows a measurement on a circular grating under angled illumination. Part (a) shows the topography of the sample. The real part of the measured field is depicted in part (c). The Fourier transform of the field is shown in part (b). The two half circles result from the convolution of the Fourier transform of the grating profile (a circle) with the Fourier transform of the projected light (a dot). This indicates that the grating modifies the incoming light field (as in normal diffraction) to create a diffracted field that can match to the plasmon resonance. Indicated by Ksp is the plasmon resonance. The excitation of the resonance is expected at an angle of $57^{\circ}$ degrees. In Part (d) only the 


\section{QMK5}

amplitude of the detected field is depicted. Several bands of waves can be seen to extend beyond the grating boundary indicating that these are propagating waves. At $57^{\circ}$ degrees a phase jump can bee seen in (c) that corresponds to the expected phase jump over the plasmon resonance.

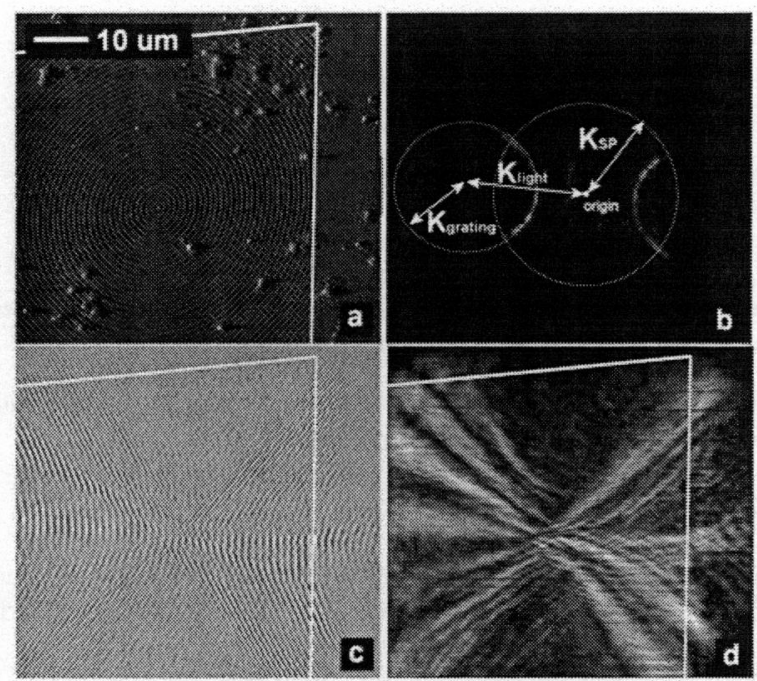

Figure 3: Circular grating, angled illumination, polarization roughly horizontal. Topography (a), measured field (c), Fourier transform showing phasematching (b), amplitude only (d). The white line indicates the border of the grating.

The grating profile in figure 4 is designed to create converging plasmons. The real part of the measured field is shown in (c), the Fourier transform in (b). The single centered ring shows the creation of converging plasmons. To highlight the fact that these are plasmons and not just diffraction, the polarization of the incoming light is tilted, which rotates the area on which the plasmons are created. Due to grating damage and residual misalignment the plasmons are mostly created on one side and do not propagate all the way to the center.

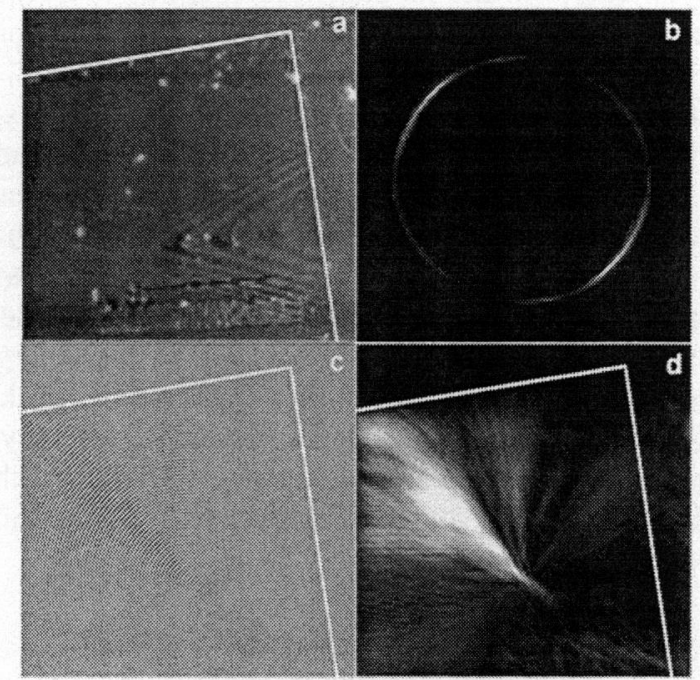

Figure 4: focusing grating for angled illumination, polarization rotated by 30 degrees. (a-d) as in figure 3 .

Plasmons created on shaped gratings like these hold promises for the creation of efficient and highly confined excitation sources at optical frequencies. The optimal shape of the gratings for focusing beyond the diffraction limit will be the subject of future research.

[1] H. Raether, Surface Plasmons (Springer-Verlag, Berlin, 1988).

[2] H.J. Lezec, A. Degiron, E. Devaux, R.A. Linke, L. Martin-Moreno, F.J. Garcia-Vidal, T.W. Ebbesen, "Beaming light from a subwavelength aperture", Science 297(5582), pp. 820-822 (2002) 